\title{
Smad4 is required for the development of cardiac and skeletal muscle in
}

\section{zebrafish}

Jie Yang*, Junnai Wang*, Zhen Zeng, Long Qiao, Liang Zhuang, Lijun Jiang, Juncheng Wei, Quanfu Ma, Mingfu Wu, Shuangmei Ye, Qinglei Gao, Ding Ma, Xiaoyuan Huang

Cancer Biology Research Centre, Tongji Hospital, Tongji Medical College, Huazhong University of Science and Technology, Wuhan, Hubei 430030, PR China

Running title: Smad4 is required for the zebrafish development

Corresponding authors: Xiaoyuan Huang, Phone: 86-27-83663351, Fax: 86-27-83663351, Email: huangxy@tjh.tjmu.edu.cn

*Note: Jie Yang and Junnai Wang contributed equally to this work

Jie Yang : yangjie@tjh.tjmu.edu.cn; Junnai Wang: 865646064@qq.com; Zhen Zeng: 304527694@qq.com; Long Qiao: 498801939@qq.com; Liang Zhuang: mrzhuangliang@126.com; Lijun Jiang: 278308418@qq.com; Juncheng Wei: 191320607@qq.com; QuanfuＭa: 99213704@qq.com; Mingfu Wu: wumingfuwh@aliyun.com; Shuangmei Ye: 29011378@qq.com; Qinglei Gao: 2482880446@qq.com; Ding Ma: dma@tjh.tjmu.edu.cn; Xiaoyuan Huang: huangxy@tjh.tjmu.edu.cn

\begin{abstract}
Transforming growth factor-beta (TGF-beta) regulates cellular functions and plays key roles in development and carcinogenesis. Smad4 is the central intracellular
\end{abstract}


mediator of TGF-beta signaling and plays crucial roles in tissue regeneration, cell differentiation, embryonic development, regulation of the immune system and tumor progression. To clarify the role of smad4 in development, we examined both the pattern of smad4 expression in zebrafish embryos and the effect of smad4 suppression on embryonic development using smad4-specific antisense morpholino-oligonucleotides. We show that smad4 is expressed in zebrafish embryos at all developmental stages examined and that embryonic knockdown of smad4 results in pericardial edema, decreased heartbeat and defects in the trunk structure. Additionally, these phenotypes were associated with abnormal expression of the two heart-chamber markers, $c m l c 2$ and $v m h c$, as well as abnormal expression of three makers of myogenic terminal differentiation, mylz2, smyhcl and mck. Furthermore, a notable increase in apoptosis was apparent in the smad4 knockdown embryos, while no obvious reduction in cell proliferation was observed. Collectively, these data suggest that smad4 plays an important role in heart and skeletal muscle development.

Keywords: Smad4, Development, Heart, Trunk, Apoptosis 
Abbreviations: smad4, SMAD family member 4; vmhc, ventricular myosin heavy chain; amhc, myh6 myosin, heavy chain 6, cardiac muscle, alpha and cmlc2, myl7 myosin, light chain 7 , regulatory;

\section{Introduction}

Transforming growth factor- $\beta$ signaling plays important roles in embryonic development and the regulation of tissue homeostasis (Moustakas and Heldin, 2009; Wu and Hill, 2009). Overactive TGF- $\beta$ signaling has been connected to fibrotic diseases and malignancies (Ikushima and Miyazono, 2010; Massague, 2008; Pardali et al., 2010). Smads play key roles in mediating TGF- $\beta$-induced programs that lead to cellular growth arrest and epithelial-to-mesenchymal transition. Smad4, a central cytoplasmic mediator of TGF signaling, was first identified as a tumor suppressor gene in pancreatic cancer by Harn et al. in 1996 and was initially designated as DPC4 (homozygously deleted in pancreatic carcinoma, locus 4) (Hahn et al., 1996). Homozygous deletion or intragenic mutation of the somatic Smad4 gene occurs frequently in colorectal, gastrointestinal, and skin carcinomas. Misregulated Smad4 expression is also commonly found in a subset of cancers (Miyaki and Kuroki, 2003; Waite and Eng, 2003). Germline mutation of Smad4 causes Juvenile Polyposis Syndrome (JPS) and targeted disruption of Smad4 in mice results in early embryonic lethality, precluding the assessment of Smad4 function in embryonic development. Yang et al. found that homozygous Smad4 Co/Co mice (chondrocyte-specific Smad4 knockout mice) suffered from severe sensorineural hearing loss (Yang et al., 2009), 
while Wang et al. showed that the deletion of Smad4 in cardiomyocytes resulted in cardiac hypertrophy characterized by an increase in the size of cardiac myocytes, age-associated fibrosis, and re-expression of certain fetal genes (Wang et al., 2005). Further, Li reported that the specific disruption of Smad4 in cerebral endothelial cells (ECs) results in perinatal $\mathrm{ICH}$ and breakdown of the blood-brain barrier (Li et al., 2011).

Ultimately, although Smad4 plays an important role in multiple biological processes, the physiological role of Smad4 in development remains poorly understood. This study investigated the expression of smad4 in zebrafish during embryonic development and found that that smad4 functions in the development of the cardiac and skeletal muscle. These findings may be useful for understanding diseases associated with heart abnormalities or skeletal muscle defects.

\section{Materials and methods}

Maintenance of zebrafish

Zebrafish were maintained under a $14-\mathrm{h}$ day/10-h night cycle at $28.5^{\circ} \mathrm{C}$. Fertilized eggs were obtained by mating adult fish from outbred colonies immediately after exposure to light. Embryos were staged according to the hours post-fertilization (hpf) and morphological criteria (Kimmel et al., 1995).

Phylogenetic analysis of smad4 protein

The sequences of smad4 protein were downloaded from the NCBI protein database. Multiple sequences alignment of smad4 proteins were performed using the ClustalX 1.83 program with default parameters (Chenna, R., 2003). To construct the 
phylogenetic tree of smad4 proteins, multiple sequences alignment using ClustalX were saved and executed by MEGA version 5.0 to generate UPGMA tree with bootstrapping analysis (Tamura, K., 2007). The phylogenetic tree was also viewed with MEGA. The node of phylogenetic tree is located between the 0.0-0.2 indicated that this protein is close between species.

RNA isolation and real-time PCR.

Total RNA was extracted from embryos or adult tissues using TRIzol solution (Life Technologies, Carlsbad, CA). The cDNA was generated from $5 \mu \mathrm{g}$ of total RNA using TOYOBO reverse transcriptase and oligo-dT primers (Invitrogen, CA, USA). The PCR reactions were performed using the cDNA as a template. The expression of smad4 and efla was analyzed using SYBR green real-time PCR primers (smad4: 5'CTGTCCTGCTAAGTTCATCTCC -3', 5' - GAGTTCAGCTCCAACCCTAATC -3', 118 bp; efla: 5'-TCAAGCCTGGTATGGTTGTGACCT-3', 5'-ACGGATGTCCTTGACAGA CACGTT-3', 149 bp). The real-time PCR analysis was performed using a CFX-96 Real-time PCR System (Bio-Rad, CA, USA).

Whole mount in situ hybridization

For in-situ hybridization, the following genes were used to generate cRNA probes: smad4, cmlc2, amhc, vmhc, mck, mylz2, smyhc1, myod, myf5 and myog. The cDNAs were amplified using RT-PCR and then cloned into the pGEM-T plasmid (TIANGEN, Beijing, CHN). Digoxigenin (DIG)-labelled RNA probes were transcribed using an RNA DIG labelling mix (Roche, BS, SW) using T7 RNA polymerase or Sp6 RNA polymerase (Ambio, TX, USA). Whole mount in situ hybridization was performed as 
previously described.

Microinjection of morpholino-oligonucleotides (MOs)

Morpholino antisense oligonucleotides (MOs) (Gene Tools, Philomath, OR, USA) were used for gene knockdown. The sequence of the wild-type MO (smad4-MO, a translation blocking morpholino) was 5'GGACATCCTGCGCTGCGTTTCACTC-3', while that of the mismatched MO (smad4-mis-MO), which contained five nucleotide mismatches, was 5'-GGAgATgCTc CGCTGCcTTTgACTC -3'. Approximately 500 pL of MOs were injected into single-cell embryos. Out of a total of approximately 60-200 MO-injected embryos, if more than $60 \%$ showed similar characteristics (not counting dead individuals) we considered these phenotypes to be consequences of the MO injection.

Calculation of heart beat rate

Embryos were then mounted laterally in $0.5 \%$ low-melting point agarose. To prevent dry out of agarose during videoing, a small aliquot of incubated embryo buffer containing tricaine was added on the top of agarose, and researchers need to sit in front of the microscope to count heartbeat frequency for 5 minutes and calculate to beats/min.

Western blotting

About 20 embryos every group were dechorionated using pronase and deyolked in Ginzburg buffer containing protease inhibitor cocktail as described (Westerfield, M., 2000). Proteins were extracted from the embryos in lysis buffer containing $1 \mathrm{mM}$ Hepes, $\mathrm{pH} 7.4,1 \mathrm{mM}$ ethylenediaminetetraacetic acid, $15 \mathrm{mM} \mathrm{NaCl}_{2}, 0.5 \%$ Triton 
X-100, Protease Inhibitor Cocktail III (Calbiochem, MA, GE) and $20 \mathrm{mM}$ $\beta$-glycerophosphate. The samples were then boiled with SDS sample buffer prior to sodium dodecylsulphate-polyacrylamide gel electrophoresis and western blotting. An anti-smad4 monoclonal antibody (BD, NJ, USA) was used as a primary antibody, and alkaline phosphatase-conjugated anti-mouse antibody IgG (Jackson ImmunoResearch, PA, USA) was used as a secondary antibody. Signals were detected using the BCIP/NBT system (Sigma, MO, USA).

Immunostaining and TUNEL assay

Zebrafish were fixed in $4 \%$ paraformaldehyde at $4{ }^{\circ} \mathrm{C}$ overnight, embedded in paraffin, and sectioned at $5 \mu \mathrm{m}$. Sections were stained with hematoxylin and eosin (H\&E). Immunostaining was performed using anti-phosphohistone $\mathrm{H} 3$ (pH3) polyclonal antibody (Eptomics, CA, USA) as a primary antibody and horseradish peroxidase-conjugated goat anti-rabbit $\operatorname{IgG}$ (Eptomics, CA, USA) as a secondary antibody, with antibody signal detected using DAB substrate (Sigma, MO, USA). The reagents for the TUNEL assay were purchased from Invitrogen (Invitrogen, CA, USA) and used according to the manufacturer's instructions.

Statistical analysis

Data from at least three independent experiments were expressed as means \pm SD and examined by one-way ANOVA followed by the Student-Newman-Keuls test. All P values were two sided. SPSS v11.5 software was used for all statistical analyses.

\section{Results}


Expression of smad4 in zebrafish development

A phylogenetic tree revealed that smad4 protein is highly conserved among a variety of vertebrates, including Mus musculus, humans, Bos taurus, Rattus norvegicus, and the lower vertebrate, zebrafish (Fig. 1A). We therefore used zebrafish as a model system to investigate the physiological role of smad4 in development in our experiments. Real-time PCR using RNA extracted from zebrafish at different developmental stages revealed that smad4 was expressed at all developmental stages, from as early as $0 \mathrm{hpf}$ up to $96 \mathrm{hpf}$. As shown in Fig. 1B, the level of smad4 mRNA increased dramatically after $0.5 \mathrm{hpf}$, peaked at $6 \mathrm{hpf}$, and then decreased sharply and was subsequently maintained at a comparatively low level from 12 to $96 \mathrm{hpf}$. In adult zebrafish, smad4 was widely expressed in the skin, gills, ovaries, heart, eyes, fins, liver, brain and swimbladder, with particularly high expression in the kidneys, trunk muscles, gallbladder, guts and testis (Fig. 1C). To visualize smad4 expression during embryogenesis in zebrafish, we performed in situ hybridization at various time points during development. As shown in Fig. 1D, smad4 expression was distributed ubiquitously at 0.2 and 8 hpf (Fig. 1D-a and b). At 17 hpf, smad4 expression was concentrated in the head and trunk muscles (Fig. 1D-c), with this expression remaining obvious until 24 hpf (Fig. 1D-d). 
A

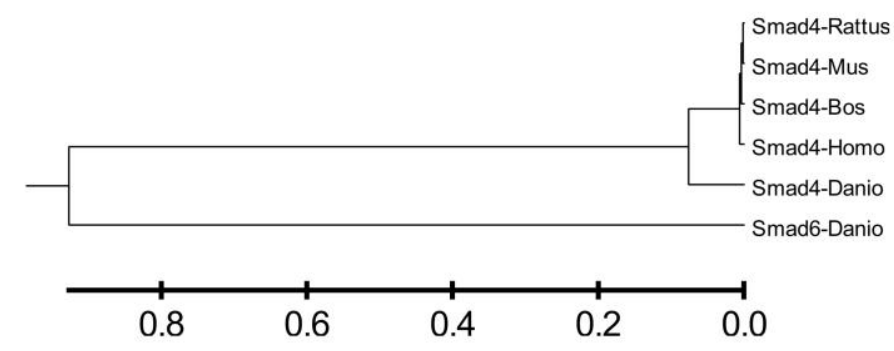

B

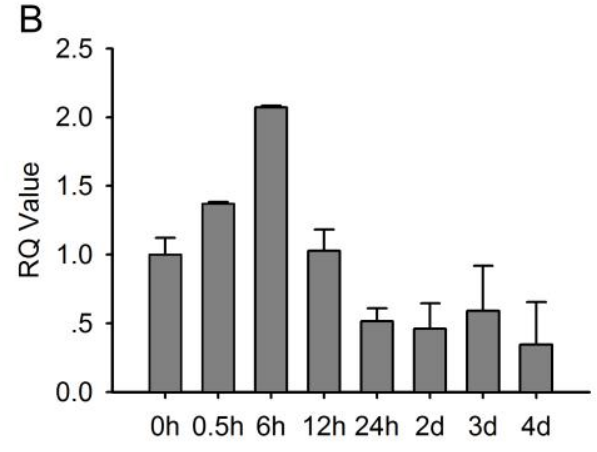

D

a

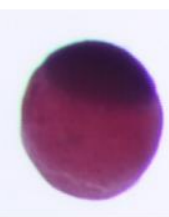

C

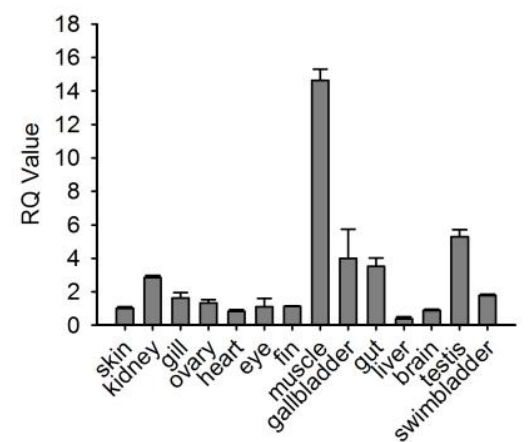

C

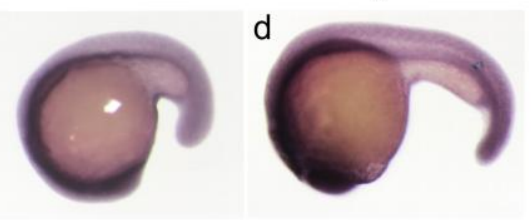

Fig. 1. Alignment of the zebrafish smad4 proteins with other vertebrates and the

expression of zebrafish smad4. (A) Phylogenetic tree showing the alignment of smad4 using protein sequences from Mus, Mus musculus; Homo, human; Bos, Bos taurus; Rattus, Rattus norvegicus; and Danio, zebrafish. Smad6- Danio was used as the out-group species. The node of phylogenetic tree is located between the 0.0-0.2 indicated that this protein is close between species. (B) RT-PCR analysis showing the expression of smad4 during embryogenesis. (C) RT-PCR analysis showing the expression of smad4 in adult tissues. (D) Expression of smad4 in zebrafish. Whole mount RNA in situ hybridization was performed using a smad4-specific antisense RNA probe and embryos at $0.2 \mathrm{hpf}$ (a), $8 \mathrm{hpf}$ (b), $17 \mathrm{hpf}$ (c), $24 \mathrm{hpf}$ (d). The blue regions indicate positive smad4 expression.

Knockdown of smad4 in developing embryos 
To examine the role of smad4 in the development of zebrafish embryos, morpholino-oligonucleotides (MOs) designed to suppress smad4 (smad4-MO) or mutant MOs (smad4-mis-MO) were injected into fertilized zebrafish eggs. The extent of smad4-MO knockdown was examined by Western blot, and significant reductions in smad4 expression with smad4-MO were observed at 24 hpf (Fig. 2A). Significantly, embryos injected with smad4-MO exhibited pericardial edema and curved trunk (Fig. 2B), which was not observed in embryos injected with smad4-mis-MO. Of note, we observed the normal number of the heart chambers (one atrium and one ventricle), but with a reduced overall size and decreased heartbeat, which lead to slower blood circulation in the morphants (Fig. 3A and C, Movie S1, Movie S2, and Movie S3). Because cardiac looping was complete at 48 hpf (Stainier, 2001), we classified heart defect severity into three grades at 72 hpf; Grade1: a mild looping defect alone; Grade2: a moderate looping defect with mild pericardial edema; Grade3: a severe looping defect with pericardial edema (Fig. 2C). Approximately $11.5 \%$ of non-injected embryos died spontaneously, indicating infertility of the embryos in our culture conditions. Injection with 3 ng of smad4-MO led to Grade2 and Grade3 defects in approximately $25 \%$ and $18 \%$ of embryos, respectively, while injection with $1.5 \mathrm{ng}$ led to Grade 2 and Grade3 defects in approximately $13.6 \%$ and $9 \%$ of embryos, respectively. This suggested that the frequency of cardiac defects increased to a significant degree $(\mathrm{p}<0.001)$ in a dose-dependent fashion (Figure 2D). Of embryos injected with 3 ng of smad4-mis-MO, Grade2 and Grade3 defects were found in 5.1\% and $2.6 \%$, respectively, indicating that Grade 2 and Grade3 heart defects are 
specifically increased $(\mathrm{p}<0.001)$ in the smad4 morphants. Analysis of trunk defects revealed that injection with $1.5 \mathrm{ng}$ and $3 \mathrm{ng}$ of smad4-MO induced a curved trunk in approximately $43 \%$ and $58 \%$ of embryos, respectively. Only $10 \%$ of embryos injected with $3 \mathrm{ng}$ of smad4-mis-MO developed this abnormality, indicating that this significant increase in curved trunk incidence $(\mathrm{p}<0.001)$ was specific to the smad4 morphants (Fig. 2E). To confirm these phenotypes, we injected zebrafish eggs with a smad4-SB-MO that suppressed normal mRNA splicing. The resulting embryos injected with smad4-SB-MO showed consistent cardiac and muscle defects, similar to those observed in the smad4-MO injected embryos (Fig.S1). These data suggest that smad4 plays a crucial role in the development of the heart and trunk.

A

B

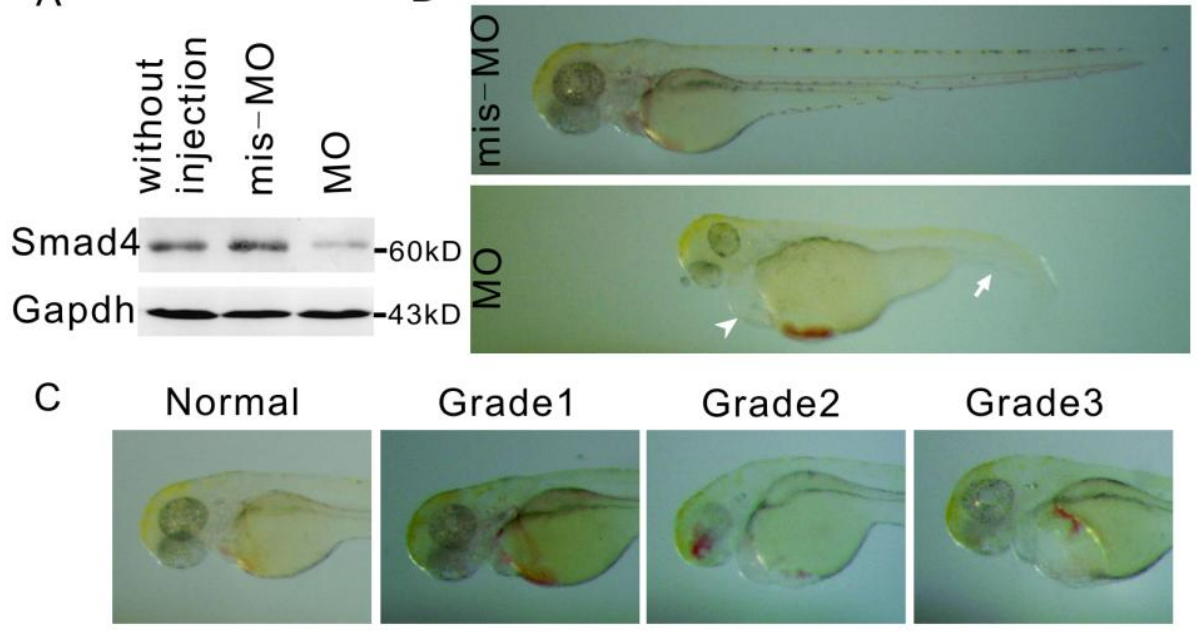

D

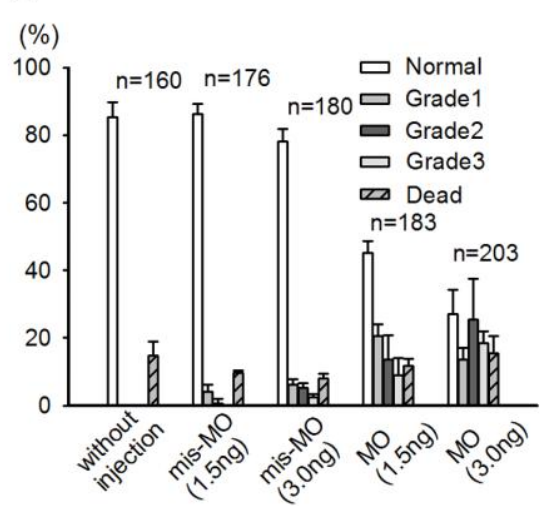

E

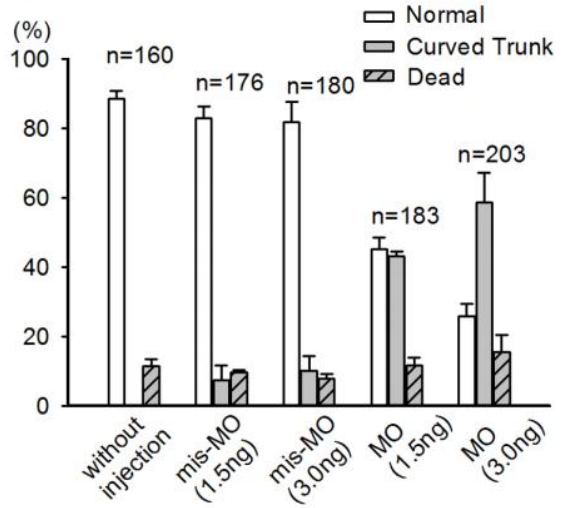


Fig. 2. Effect of smad4 knockdown in zebrafish embryos using smad4-MO or smad4 mis-MO.

(A) Western blot analysis of smad4 expression in uninjected and smad4-MO-injected embryos. (B)

Phenotype of embryos injected with smad4-MO or smad4-mis-MO at $72 \mathrm{hpf}$. The pericardial

edema (arrow head) and curved trunk (arrow) were observed in smad4 morphants. (C)

Morphological classification of heart defects. The degree of cardiac defect in the morphants was

classified into three grades at 72 hpf. Normal: Normal heart. Grade1: Heart shows abnormality

with mild looping defect and pericardial edema; Grade2: Heart shows abnormality with moderate

looping and defect and pericardial edema; Grade3: Heart shows abnormality with string-like heart

with severe pericardial edema. Embryos are shown in lateral view. (D) Frequencies of heart defects in embryos injected with 1.5 or $3.0 \mathrm{ng}$ of smad4-mis-MO and 1.5 or $3.0 \mathrm{ng}$ smad4-MO, respectively at 48 hpf. (E) Frequencies of curved trunk in embryos injected with 1.5 or $3.0 \mathrm{ng}$ of smad4-mis-MO and 1.5 or $3.0 \mathrm{ng}$ smad4-MO, respectively at $48 \mathrm{hpf}$.

Expression of cardiac markers in smad4 morphants

To further investigate the mechanism(s) underlying the heart defects observed in the smad4 morphants, we examined the expression of seven markers, four anterior lateral plate mesoderm (ALPM) markers including GATA-binding protein 5 (gata5), stem cell leukemia protein $(s c l)$, NK2 transcription factor related $5(n k x 2.5)$, and heart and neural crest derivatives expressed transcript2 (hand2), and three cardiac chamber markers including ventricular myosin heavy chain $(v m h c)$, atrial myosin heavy chain $(a m h c)$ and cardiac myosin light chain2(cmlc2), which are expressed in the heart tube of zebrafish embryos at $24 \mathrm{hpf}$ and are specific to ventricle, atrium, and both chambers, respectively (Stainier, 2001). In situ hybridization demonstrated that, at 12 
hpf, the expression of gata5, scl, $n k x 2.5$ and hand 2 in smad4 morphants was similar to that in the control embryos injected with smad4-mis-MO or without injection (Fig. S2), suggesting that smad4 is not involved in the early myocardial specification ; at 30 hpf, the expression of amhc was identical in both the controls and the morphants. The expression of $c m l c 2$ and $v m h c$ was slightly decreased in the morphants compared to the control embryos (Fig. 3B), mean the number of $c m l c 2+$ and $v m h c+$ cells decrease, potentially explaining the abnormal elongation of the heart tube in the morphant embryos (Stainier, 2001). Analysis of stained sections revealed a decrease in the overall size of the pericardium, atrium and ventricle, with a simultaneous increase in the thickness of the pericardial and both chamber walls in morphants at $120 \mathrm{hpf}$. Besides, the cardiac A-P pattern is failed to occur in the morphants, so the orientation of both chambers are not right. These findings indicate that cardiac defects in smad4 morphants may result from impaired maturation, delayed development of cardiomyocytes and the failure conversion of the chambers.

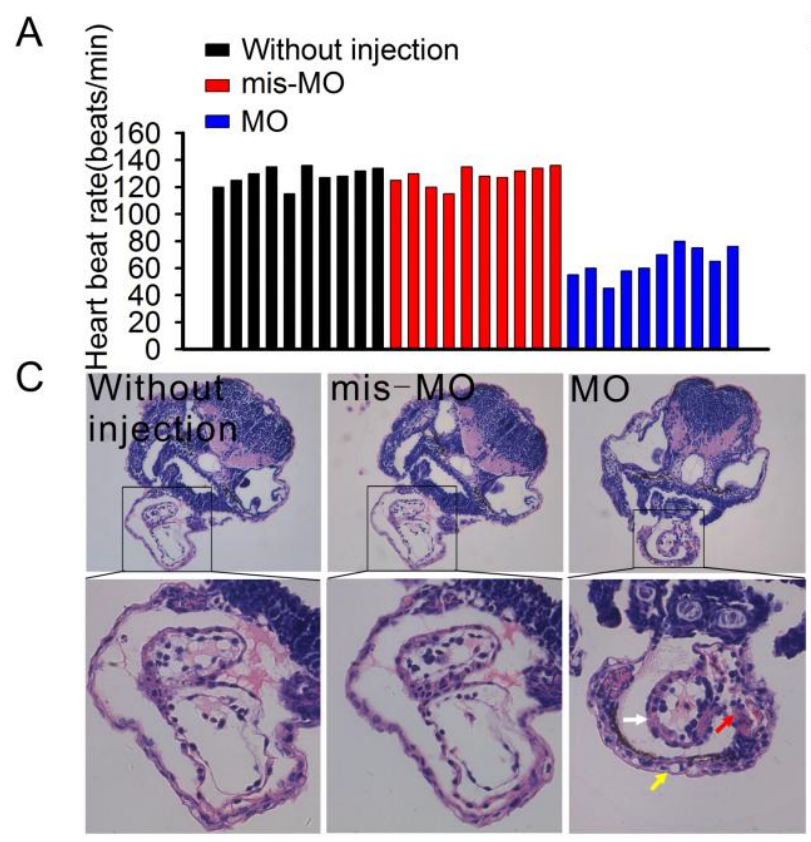

B

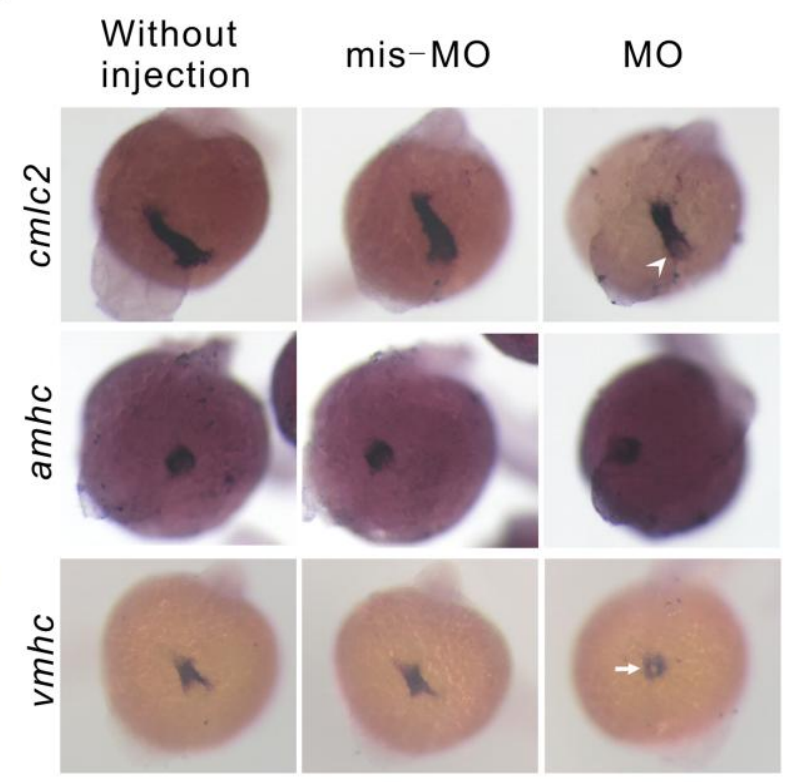


Fig. 3. Heart defect of smad4 knockdown zebrafish. (A) Heartbeats of the control and morphant embryos at 48 hpf. (B) Expression of $v m h c, a m h c$, and $c m l c 2$ in the control and morphant embryos at 24 hpf. Arrowhead indicates abnormal $c m l c 2$ expression in the whole heart. Arrow indicates abnormal $v m h c$ expression in the ventricle. Embryos are shown in dorsal view, anterior oriented downward. (C) H\&E staining was performed on heart sections from the control and morphant embryos at $120 \mathrm{hpf}$. White, red and yellow arrows indicate abnormal structures in the ventricle, atrium and pericardium.

Expression of myogenic markers in Smad4 morphants

Six markers were examined to clarify the mechanism(s) underlying curved trunks. Skeletal muscle myosin light polypeptide 2 (mylz2), slow myosin heavy chain 1 (smyhcl), and muscle creatine kinase ( $m c k$ ) were used to clearly identify terminally differentiated skeletal muscle, along with the myogenic regulatory factors myogenic differentiation (myod), myogenic factor 5 (myf5) and myogenin (myog). Mylz2, smyhcl, and mck were used as markers to differentiate between fast muscle, slow muscle, and both slow and fast muscles, respectively (Xu et al., 2000). Myod and myf5 were found to be expressed in the two lines of adaxial cells lying adjacent to the somites of the notochord, while myog was also found in these two lines of cells as well as in the paraxial mesoderm at $12 \mathrm{hpf}$. In situ hybridization clearly showed that the expression of myod, myog and myf5 was unchanged between the morphants and the controls at $24 \mathrm{hpf}$ (Fig. 4B). However, at $24 \mathrm{hpf}$, when skeletal muscle differentiation was complete, the expression patterns of mylz2, smyhcl and mck in the morphants were distinct from those in either the uninjected control embryos or those 
injected with smad4-mis-MO (Fig. 4A). These data suggest that the abnormal trunk morphology observed in the smad4 morphants is not caused by the perturbation of myogenic regulatory factors, but is most likely the result of increased expression of muscle differentiation factors such as mylz2, smyhcl and $m c k$.
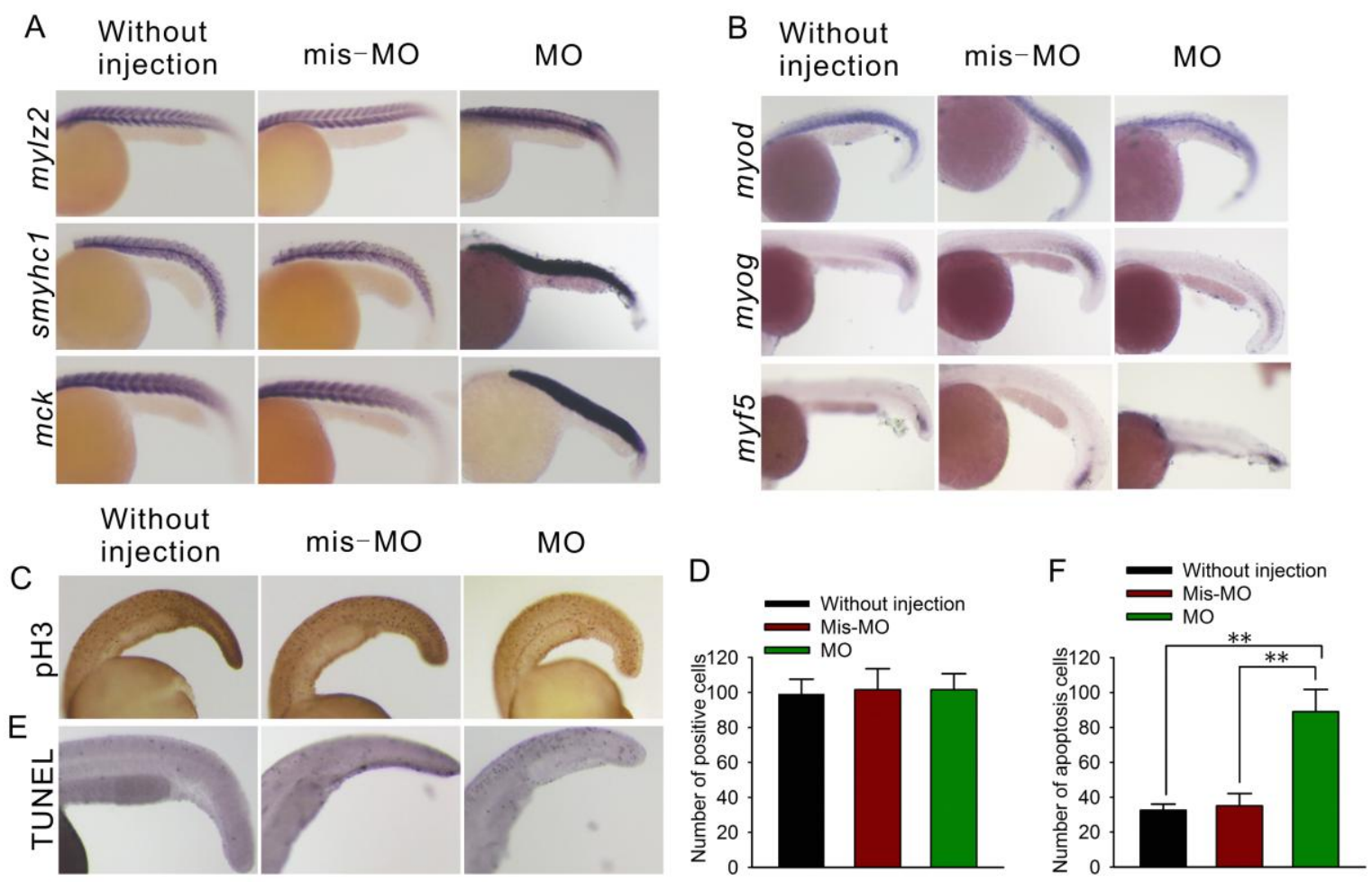

Fig. 4. Changes to the trunk in smad4 knockdown zebrafish. (A) Expression of mylz2, smyhcl, and $m c k$ in the control and morphant embryos at 24 hpf. (B) Expression of myod, myog, and myf5 in the control and morphant embryos at $24 \mathrm{hpf}$. (C) Staining of pH3-positive cells in control and morphant embryos. (D) Quantification of $\mathrm{pH} 3$ positive cells. (E) Analysis of cell death using a TUNEL assay in control and morphant embryos. (F) Quantification of apoptotic cells. Five embryos were counted for each condition; the error bars indicate the standard deviation based on five samples. $\mathrm{pH} 3$ staining $\mathrm{p}=0.84$; TUNEL assay, at $24 \mathrm{hpf}, * * \mathrm{p}<0.01$.

Although the trunk underwent at least some level of development in smad4 morphant embryos, overall trunk shape was severely affected, as indicated by the 
expression of specific marker genes. We next asked whether changes in cell death or cell proliferation correlated with these developmental defects in morphant embryos. Immunostaining with anti-phosphorylated histone $3(\mathrm{pH} 3)$ antibody, which labels mitotic cells, was performed to assess differences in cell proliferation in the trunk between morphants and control embryos. No obvious differences were observed among these embryos, indicating that the curved trunk in smad4 morphants was not a consequence of altered cell proliferation (Fig. C and D). Apoptotic cells in morphants and control embryos were identified using a TUNEL assay, with the number of positive cells were being greatly increased in the morphants (Fig. 4E). The differences between the morphants and control embryos were highly significant $(\mathrm{p}<0.01)$ (Fig. 4F), leading us to conclude that apoptosis of cells in the trunk is a major effect of smad4-MO in zebrafish embryos.

\section{Discussion}

Recent studies have revealed that SMAD proteins are involved in the development of cardiac and skeletal muscle. For example, Smad1 and smad5 act downstream of BMP-2 signaling, which inhibits myogenic differentiation and induces

osteoblast differentiation in $\mathrm{C} 2 \mathrm{C} 12$ myoblasts (Yamamoto et al., 1997). Co-overexpression of Smad1, a ligand-specific Smad, and Smad4, a common Smad, restored the ability of P19CL6 cells to differentiate into cardiomyocytes, whereas stable overexpression of Smad6, an inhibitory Smad, completely blocked differentiation of P19CL6 cells, suggesting that the Smad pathway is necessary for cardiomyocyte differentiation (Monzen et al., 2001). Smad6 inhibits BMP-mediated 
SMAD activation (de Pater et al., 2012; Ishisaki et al., 1999), and while overexpression of Smad6 prevents myocyte differentiation, Smad6 knockout mice display several cardiovascular abnormalities (Galvin et al., 2000). Smad7, which is also an inhibitory SMAD protein, cooperates with MyoD to create a positive loop that induces Smad7 expression and promotes MyoD-driven myogenesis (Kollias et al., 2006). Moreover, Smad7 plays a protective role in Ang II-induced cardiac remodeling via the Sp1-TGF-beta/Smad-NF-kappaB-miR-29 regulatory network. Endothelial-specific knockout of Smad4 resulted in loss of valve-forming activity, and the Smad4-deficient endocardium was associated with diminished epithelial-to-mesenchymal transition, reduced endocardial proliferation, and loss of Id2 expression in the valve-forming regions (Wei et al., 2013). In addition to these reports, we have shown here that smad4 plays an important role in the development of cardiac and skeletal muscle in zebrafish.

In this study, we demonstrate that smad4 is expressed both during zebrafish embryogenesis and in adult zebrafish, which is consistent with previous research (Dick et al., 2000), and we show that knockdown of zebrafish Smad4 results in abnormal looping of the heart tube accompanied by pericardial edema and decreased heartbeat. Heart development is governed by a complex gene regulatory network consisting of transcription factors, their co-factors, and downstream genes modulating cell fate specification, cellular differentiation, cell proliferation, and cell migration. The expression patterns of $c m l c 2$ and $v m h c$, as revealed by in situ hybridization, illustrate the restricted reorganization of myocardiocytes during cardiac fusion and 
heart tube assembly in smad4 morphants. However, the expression of amhc was normal in these embryos at $24 \mathrm{hpf}$. In addition, tissue sections revealed that heart size in the smad4 morphants was decreased relative to the control, with abnormal positioning of the cardiac chambers compared to the control. These data imply that smad4 is not only involved in regulating early growth and migration of cardiomyocytes, but may also regulate chamber shape, relocation and morphology. Surprisingly, heart formation and morphogenesis were not obviously affected in cardiomyocyte-specific Smad4 knockout mice. This finding suggests that Smad4 might not be the sole mediator of all TGF- $\beta$ signals in cardiac myocytes. Bmp signaling there are 3 receptor-Smad proteins (Smad1, -5, and -8) that can be phosphorylated and interact with the co-Smad, Smad4. This phosphorylated Smad-protein complex is translocated to the nucleus where it activates gene expression. De Pater demonstrated that smad6 is required to inhibit Bmp signaling and that the downregulation of Bmp signaling is required for proper formation of the ventricle and preventing expression of atrial myosin in regions outside the atrium(de Pater et al., 2012). Knockdown of smad4 may disturb the BMP signaling pathway and induce heat malformation, and the above results provide a new angle to understand the signaling pathway about heart development.

In addition to heart defects, we show that smad4 morphants develop a curved trunk, which is associated with overexpression of mylz2, smyhcl and mck at $24 \mathrm{hpf}$. Casari's team also found this phenomenon, which supports our finding (Casari et al., 2014 ). Trunk skeletal muscle in vertebrates originates from the primary myotome of 
the somites. The molecular mechanisms governing muscle cell differentiation have been well characterized (Molkentin and Olson, 1996; Olson and Klein, 1994). Two classes of transcription factors play a crucial role in the process: the myogenic basic helix-loop-helix proteins (including Myod, Myog, Myf5, and MRF4) and members of the MEF2 (myocyte-specific enhancer factor 2) family. Terminal differentiation of muscle cells results in the expression of a set of MSP genes, including mylz2, smyhc1 and mck. As both MEF2 and myogenic bHLH transcription factors are directly involved in the activation of these MSP genes (Kaushal et al., 1994; Molkentin et al., 1995), the overexpression of these three genes in smad4 morphants may be the result of deregulation of MEF2 and MRF4. However, other mechanisms of MSP regulation may also be involved, because the expression of myod, myog and myf5 were unchanged between smad4 morphants and controls. For example, Yuhua Sun demonstrated that MGA/Max/Smad4 act in the yolk syncytial layer to reinforce ventralization via BMP signaling pathway, and knockdown the Smad4 by Smad4-MO, Smad4-MO morphants lacked the ventral tailfin(Sun et al., 2014). That supported our study. From this data, it is tempting to speculate that smad4 might also regulate the expression of MSP genes through BMP signaling. Although additional studies are needed to clarify the mechanism(s) by which smad4 influences muscle development, our findings should serve as a starting point for elucidating the role of smad4 in myogenesis.

Although smad4 morphants displayed cardiac defects and a curved trunk, cardiac and skeletal myogenesis seem to be normal in the early stages of embryonic development. 
Therefore, smad4 may not be involved in cell specification or differentiation, but instead in the maturation or proliferation of differentiated myogenic cells. Resident progenitor cell populations, which are essential for muscle growth as development proceeds, maintain the balance between self-renewal and differentiation. Studies have shown that activation of SMADs by TGF- $\beta$ family members results in fibrotic, apoptotic, and anti-hypertrophic processes that are related to detrimental cardiac remodeling and progression to heart failure (Euler-Taimor and Heger, 2006). Smad4 was originally considered to act as a co-SMAD protein in the TGF- $\beta$ signal transduction pathway, and knockdown of its expression might be expected to block apoptosis. However, we observed that the loss of smad4 function resulted in an obvious increase in the number of apoptotic cells in the curved trunk of zebrafish embryos. It may be that the loss of smad4 function leads to the failure of skeletal progenitor cells to differentiate, leading to muscle apoptosis.

Taken together, we have shown that smad4 plays a pivotal role in cardiac and skeletal muscle development. These findings will help elucidate the molecular mechanisms underlying the development of heart and skeletal muscle.

\section{Acknowledgements}

This work was funded by grant from National Science Foundation of China (30901587, 81201639, 2015CB553903, 2015BAI13B05), and Tongji Hospital (2201101877). 